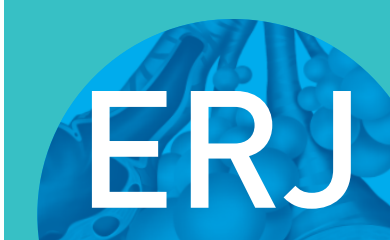

open research
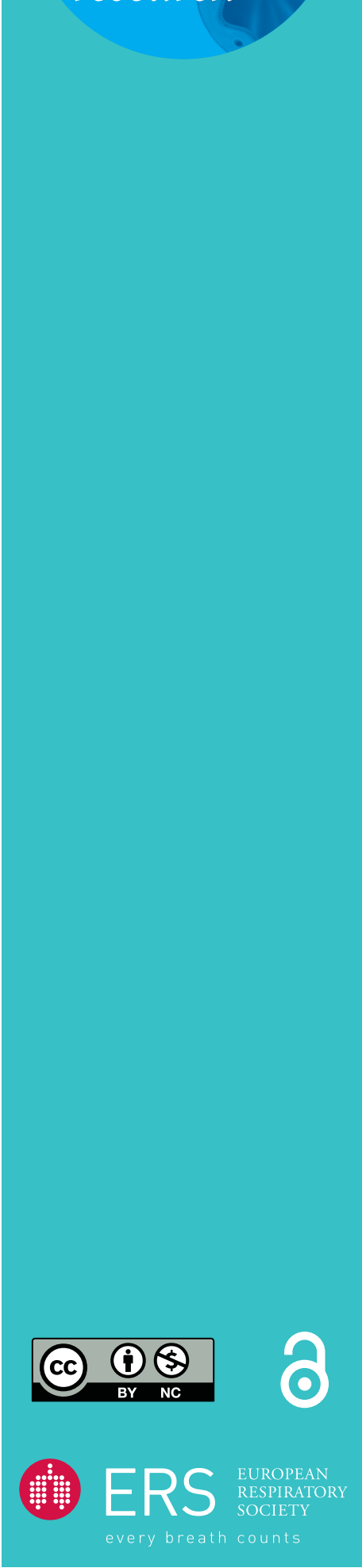

\section{Intrinsic factors influence self-management participation in COPD: effects on self-efficacy}

To the Editor:

Chronic Obstructive Pulmonary Disease (COPD) is a leading cause of morbidity worldwide. Lifestyle changes (e.g. smoking cessation, dietary interventions and physical activity) are recommended to decelerate disease progression and improve quality of life. Adequate self-management can facilitate these changes [1]. Although self-management programmes (SMPs) may be an effective tool to reduce morbidity and burden in chronic diseases [2], their contribution to COPD treatment remains controversial [3, 4]. For example, not all patients are suitable to participate in SMPs and it remains unclear why patients actually participate in SMPs [2]. Although disease severity may be an important reason for patients to participate in an SMP [5], it is unlikely to be the sole explanation. We hypothesised that disease-independent personal traits will influence coping behaviour and subsequent participation in SMPs. Therefore, we compared "wish for self-efficacy", "mastery" and "readiness to change" between users and nonusers of an SMP in regular COPD care.

Users and nonusers of an online SMP (www.MijnCOPDonline.nl; Curavista bv, Geertruidenberg, the Netherlands) integrated into the routine care of two teaching hospitals in Rotterdam, the Netherlands, were invited to participate. MijnCOPDonline allows patients to monitor their disease, exacerbations, treatment goals and medication, and contact their healthcare provider via an electronic consultation. All participants had a diagnosis of COPD confirmed by a pulmonologist. Users and nonusers of this online SMP were invited to participate in this study. Illiterate patients and patients with a current malignancy were excluded from the study. Nonusers were randomly drawn from a list of patient numbers. All eligible users and nonusers were invited to participate: the first invitation, which was accompanied by a first set of surveys, were sent out by post in January $2016(t 0)$. Participants received a second set of surveys in December $2016(t 1)$. The following validated surveys were used: 1) Clinical COPD Questionnaire (CCQ), reflecting health status in COPD; 2) modified Medical Research Council scale (mMRC), reflecting dyspnoea; 3) Partners In Health (PIH-NL) [6], reflecting a wish for self-efficacy and mastery; 4) Patient Activation Measure (PAM13-NL) [7], reflecting readiness to change; 5) Short Self-Management Ability Scale (SMAS-s) [8]; and 6) Euroquol-5D3L (EQ-5D3L) [9], reflecting patient's general health status. An additional survey asked about gender, smoking status, educational level and exacerbation frequency in the previous year.

Differences in characteristics between users and nonusers were tested with Chi-squared tests, unpaired Student's t-tests and Mann-Whitney U-tests, where appropriate.

Adjusted differences in primary and secondary measures between users and nonusers were evaluated using multiple linear regression analysis adjusted for age, gender and disease-defining parameters according to the Global Initiative for Chronic Obstructive Lung Disease (GOLD) criteria A-D (airflow limitation (forced expiratory volume in $1 \mathrm{~s}$ (FEV1) \% of predicted), exacerbation frequency, mMRC and CCQ) [10].

The medical research ethics committee declared this study to be outside the scope of the Medical Research Involving Human Subjects Act and that no formal approval or informed consent was required.

@ERSpublications

Personal traits should be addressed before offering self-management programmes to patients with COPD http://ow.ly/DtNh30k1A15

Cite this article as: de Boer GM, Mennema TH, van Noort E, et al. Intrinsic factors influence self-management participation in COPD: effects on self-efficacy. ERJ Open Res 2018; 4: 00011-2018 [https://doi.org/10.1183/23120541.00011-2018].

Copyright $\odot$ ERS 2018. This article is open access and distributed under the terms of the Creative Commons Attribution NonCommercial Licence 4.0. 
Of the 78 users and 316 nonusers of the online SMP that were invited, 43 users and 132 nonusers participated at $t 0(44.4 \%, 95 \%$ CI $39.4-49.5 \%)$, and 39 users and 92 nonusers at $t$. Of all participants, $35.1 \%$ were nonresponders, and three died between to and $t 1$. Users of an SMP were more likely to participate $(\mathrm{p}=0.05$ at $t 0$ and $\mathrm{p}=0.004$ at $t 1)$. In both rounds, users were slightly younger than nonusers $\left(\mathrm{p}=0.006\right.$ at $t 0$ and $\mathrm{p}=0.004$ at $\left.t_{1}\right)$. Although users at $t_{0}$ were more frequently women, this difference had disappeared at $t_{1}(\mathrm{p}=0.025$ versus $\mathrm{p}=0.10)$. In both rounds, no difference was found for disease burden, lung function or GOLD criteria A-D. Educational level was comparable at $t_{0}$ and $t 1(\mathrm{p}=0.32)$. At $t 0$, users encountered more exacerbations than nonusers (median 2 versus $1, \mathrm{p}=0.018$ ); however, this difference had disappeared at $t 1$ (median 1 versus $1, \mathrm{p}=0.56$ ).

On PAM13-NL, users scored significantly higher than nonusers at to (mean 57.13 versus $51.54, \mathrm{p}=0.03$ ) and at $t 1$ (mean 58.11 versus 51.52, $\mathrm{p}=0.026$ ); the mean PAM13-NL scores remained significantly different after adjustment for covariables ( $t 0$ : adjusted $\left.\mathrm{R}^{2}\left(\mathrm{aR}^{2}\right)=0.08, \mathrm{p}=0.004 ; t 1: \mathrm{R}^{2}=0.19, \mathrm{p}=0.004\right)$. This was also the case for PIH-NL ( $t 0$ : mean 79.7 versus $72.3, \mathrm{p}=0.001$; $t 1$ : mean 82.0 versus $74.0, \mathrm{p}<0.001$ ) (figure 1 ) before and after adjustment $\left(t 0: \mathrm{aR}^{2}=0.09, \mathrm{p}=0.002 ; t 1: \mathrm{aR}^{2}=0.009, \mathrm{p}<0.001\right)$. Mean EQ-5D3L and SMAS-s scores were not significantly different between $t 0$ and $t$. However, at $t$, for the user group only, there was a significant improvement seen on SMAS-s after adjustment for covariables $\left(a R^{2}=0.13, p=0.022\right)$.

This is the first study to demonstrate that COPD patients using an online SMP have a higher wish for self-efficacy and mastery and are more inclined to change than nonusers. Users of an SMP had higher PIH-NL and PAM13-NL scores, even after adjustment for age, gender and disease-defining parameters (airflow limitation (FEV $1 \%$ of predicted), exacerbation frequency, mMRC and CCQ). This implies that these disease-independent personal factors play an important role in participation in online SMPs. The mean PAM13-NL score was $\geqslant 5$ points higher among the SMP users, both at $t_{0}$ and $t$, indicating a clinically relevant difference [7].

In addition, we are the first to demonstrate that COPD patients using this online SMP in routine care improved their self-efficacy over time. After adjustments for age, gender and disease severity, an improvement was noticed on SMAS-s scores. Self-efficacy is described as how well one can execute courses of action required to deal with expected future situations [11]. An improvement in self-efficacy could therefore been seen as a relevant improvement for COPD patients in real life. Unlike previous studies, disease characteristics and educational level did not affect participation in the online SMP [12]. This strengthens our finding that personal intrinsic factors are probably more important in the use of an online SMP than other factors; also, the use of an online SMP seems to improve self-management abilities. Although survey studies are subject to response bias, the nonresponse rate in this study was relatively low. However, since we cannot exclude that the online SMP was not offered to all COPD patients, selection bias might have occurred; perhaps only patients who were pursuing self-efficacy and mastery, or were more inclined to change, participated in the SMP. Although we do not know the reasons why patients did not accept our invitation to participate, these general points might even further strengthen our findings.

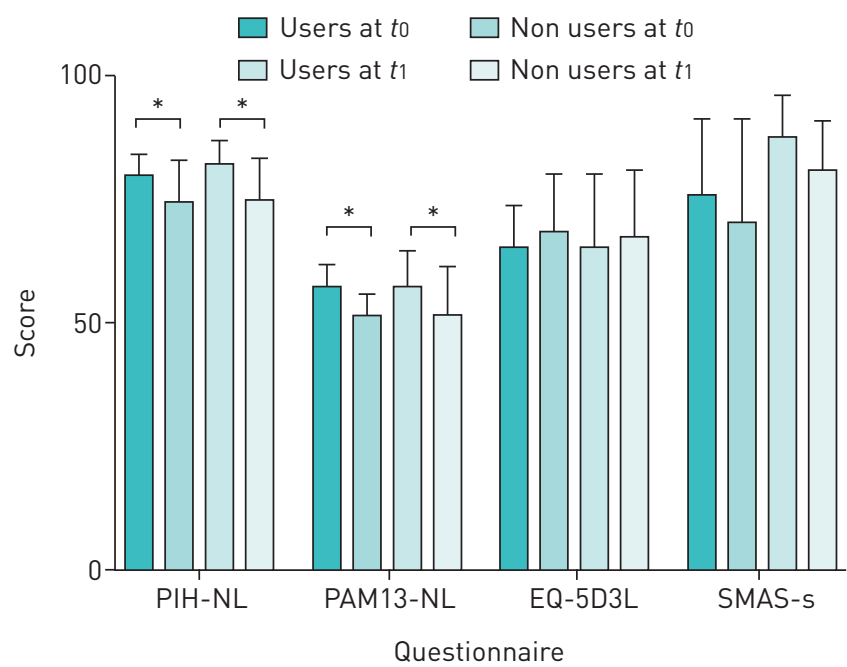

FIGURE 1 Survey scores for Partners In Health (PIH-NL), Patient Activation Measure (PAM13-NL), Euroquol-5D3L (EQ-5D3L) and Short Self-Management Ability Scale (SMAS-s) of users and non-users adjusted for forced expiratory volume in $1 \mathrm{~s} \%$ of predicted, Clinical COPD Questionnaire, modified Medical Research Council score, exacerbation frequency, age and gender (with p-values). EQ-5D3L utility scores were rescaled to $0-100$ scores. *: $p<0.05$. 
This observational study is the first step to unravelling the conflicting evidence on the use of SMPs. This study was not randomised and we have no data on the patients' status before the SMP was implemented. Nevertheless, our results indicate that disease-independent personal factors are central in the use of an online SMP in routine care and should be taken into consideration in future research.

In conclusion, coping strategies might improve under the influence of a self-management programme. In addition, intrinsic factors, such as self-efficacy, mastery and readiness to change, are targets involved in the aim to optimise the routine care offered by SMPs. In particular, a patient's readiness to change and their wish for self-efficacy should be addressed before offering an SMP, regardless of disease severity.

Geertje M. de Boer ${ }^{1}$, T.H. Mennema ${ }^{2}$, Esther van Noort ${ }^{3}$, Niels H. Chavannes ${ }^{4}$, Erwin Birnie ${ }^{5,6}$ and Johannes C.C.M. in 't Veen ${ }^{1}$

${ }^{1}$ Dept of Pulmonology, Franciscus Gasthuis en Vlietland, Rotterdam, The Netherlands. ${ }^{2}$ Dept of Pulmonology, Havenziekenhuis, Rotterdam, The Netherlands. ${ }^{3}$ Curavista bv, Geertruidenberg, The Netherlands. ${ }^{4}$ Dept of Public Health and Primary Care, Leiden University, Leiden, The Netherlands. ${ }^{5}$ Dept of Statistics and Education, Franciscus Gasthuis en Vlietland, Rotterdam, The Netherlands. ${ }^{6}$ Dept of Obstetrics and Gynaecology, University Medical Center Utrecht, Utrecht University, Utrecht, The Netherlands.

Correspondence: Geertje Maria de Boer, Dept of Pulmonology, Franciscus Gasthuis en Vlietland, Kleiweg 500, 3045PM Rotterdam, The Netherlands. E-mail: G.Boer3@franciscus.nl

Received: Jan 182018 | Accepted after revision: April 272018

Author contributions: G.M. de Boer is the guarantor of the content of the manuscript, including the data and analysis. J.C.C.M. in 't Veen, T.H. Mennema and G.M. de Boer were responsible for the study design. Data collection was mainly performed by G.M. de Boer with help from E. van Noort. E. Birnie and G.M. de Boer were responsible for statistical analysis. G.M. de Boer was responsible for the first draft of the manuscript. J.C.C.M. in 't Veen, T.H. Mennema, E. Birnie and N.H. Chavannes critically reviewed the manuscript.

Conflict of interest: T.H. Mennema reports receiving an unrestricted grant to her faculty from AstraZeneca, the Netherlands, for the conduct of the study. G.M. de Boer reports receiving an unrestricted grant to her faculty from AstraZeneca, the Netherlands, for the conduct of the study J.C.C.M. in 't Veen reports receiving grants from AstraZeneca during the conduct of the study.

Support statement: AstraZeneca, The Netherlands, funded this research with an unrestricted grant. Funding information for this article has been deposited with the Crossref Funder Registry.

\section{References}

1 Trappenburg J, Jonkman N, Jaarsma T, et al. Self-management: one size does not fit all. Patient Educ Couns 2013; 92: $134-137$.

2 Kruis AL, van Schayck OC, in't Veen JC, et al. Successful patient self-management of COPD requires hands-on guidance. Lancet Respir Med 2013; 1: 670-672.

3 Bischoff EW, Hamd DH, Sedeno M, et al. Effects of written action plan adherence on COPD exacerbation recovery. Thorax 2011; 66: 26-31.

4 Fan VS, Gaziano JM, Lew R, et al. A comprehensive care management program to prevent chronic obstructive pulmonary disease hospitalizations: a randomized, controlled trial. Ann Intern Med 2012; 156: 673-683.

5 Effing T, et al. Self-management education for patients with chronic obstructive pulmonary disease. Cochrane Database Syst Rev 2007; 4: CD002990.

6 Petkov J, Harvey P, Battersby M. The internal consistency and construct validity of the Partners in Health scale: validation of a patient rated chronic condition self-management measure. Qual Life Res 2010; 19: 1079-1085.

7 Fowles JB, Terry P, Xi M, et al. Measuring self-management of patients' and employees' health: further validation of the Patient Activation Measure (PAM) based on its relation to employee characteristics. Patient Educ Couns 2009; 77: 116-122.

8 Cramm JM, Strating MM, de Vreede PL, et al. Validation of the Self-Management Ability Scale (SMAS) and development and validation of a Shorter Scale (SMAS-S) among older patients shortly after hospitalisation. Health Qual Life Outcomes 2012; 10: 9.

9 Johnson JA, Luo N, Shaw JW, et al. Valuations of EQ-5D health states: are the United States and United Kingdom different? Med Care 2005; 43: 221-228.

10 Global Initiative for Chronic Obstructive Lung Disease. Global Strategy for the Diagnosis, Management and Prevention of Chronic Obstructive Pulmonary Disease. http://goldcopd.org/wp-content/uploads/2017/11/GOLD2018-v6.0-FINAL-revised-20-Nov_WMS.pdf.

11 Bandura A. Self-efficacy mechanism in human agency. Am Psychol 1982; 37: 122-147.

12 Dalgard OS, Mykletun A, Rognerud M, et al. Education, sense of mastery and mental health: results from a nation wide health monitoring study in Norway. BMC Psychiatry 2007; 7: 20. 